\title{
Sense of Learning Achievement for Excretion Assistance Techniques Through on-campus Training and on-site Clinical Training for Nursing Students
}

\begin{abstract}
Aoki Noriko*
The Jikei University School of Nursing, Chofu-City kokuryo, Tokyo, Japan.

"Correspondence:

Aoki Noriko, The Jikei University School of Nursing, 182-8570

Chofu-City kokuryo8-3-1 Tokyo Japan, Fax: 81-03-3488-7452;

Tel: 81-03-3480-1151; E-mail: noriko_a@jikei.ac.jp.

Received: 04 September 2018; Accepted: 30 September 2018

Citation: Aoki Noriko. Sense of Learning Achievement for Excretion Assistance Techniques Through on-campus Training and on-site Clinical Training for Nursing Students. Nur Primary Care. 2018; 2(5): 1-7.
\end{abstract}

\section{ABSTRACT}

Objectives: This study was to understand which nursing skills are easy to learn, and which are not at on-campus and on-site clinical training by conducting a questionnaire survey on the sense of learning achievement regarding excretion assistance techniques on-campus and on-site clinical training on excretion for junior nursing students learning such nursing techniques for the first time.

Methods: We conducted a self-administered, anonymous questionnaire surveys a total of 3 times, using our uniquelydeveloped questionnaire form. The survayl was the assistance techniques for genital care and diaper changing. The survay 2 was conducted after the 2 nd grade students learned assistance techniques for temporary urinary catheterization. The survay 3 was conducted after the 2 nd grade students completed on-site clinical training.

Results: The highest sense of learning achievement; the survayl was "understanding of the necessity and significance of assistance", followed by "acquisition of knowledge, the survay2 was "understanding the necessity and significance of assistance" and "acquisition of knowledge", and the survya3 was "understanding of the relationship with existing knowledge", followed by "attention to safety". The lowest sense of learning achievement; the survay1 was "implementation of assistance", followed by "understanding of the patient's response, the survay 2 was "evaluation of the results of assistance", followed by "understanding of the patient's response, and the survay 3 was "understanding of the patient's thoughts and their application in the practice of assistance", followed by "assistance with understanding the patient's response".

Discussion: Regarding the learning achievement for excretion assistance, it was found that it was easy for students to understand the knowledge and significance of excretion assistance and to pay attention to safety. It is shown that students felt that it was more difficult to consider privacy, provide comfortable assistance, and implement assistance with an understanding of patient thoughts during on-site clinical training, compared to on-campus training.

\section{Keywords}

Excretion assistance techniques, Nursing skill, The sense of learning achievement, Acquire Questionnaire Survey.

\section{Introduction}

Background of Research

Excretion assistance involves unique difficulties accompanying excretion, including securing privacy in response to patients' embarrassment as well as paying attention to their self-respect. Accordingly, it is difficult for nursing students who take charge of patients for the first time to fully acquire excretion assistance techniques. Just before their graduation, nursing students indicate that their subjective sense of achievement regarding excretion assistance techniques other than diaper changing and genital care is less than $50 \%$ and they are unable to master sufficient skills under the present circumstance [1]. Among the acquired nursing 
techniques of novice nurses at the time of employment, fewer of those novice nurses stated that they were able to perform excretion assistance independently, relative to meal care and hygienic care. While more of them answered that they had experience administering excretion assistance, they also had little confidence providing such assistance, and most of them only had experience of watching the procedures for bladder catheter insertion, enema administration, and stool extraction [2].

One reason for the difficulty in acquiring excretion assistance techniques may be due to difficulties in implementing excretion assistance as well as securing privacy in response to patients' embarrassment and sexuality issues. When on-campus training for excretion assistance is implemented at our university, students play both patient and nurse roles with one another. During such training, genital areas and other bare skin is covered to secure privacy. Therefore, most students see and touch the genital areas of others for the first time while providing excretion assistance, such as genital care and diaper changing at on-site clinical training. They appeared uncertain regarding the degree of intervention to take, as well as how to talk to the patients. Nursing students exhibited a sense of embarrassment, tension and strong hesitation during on-campus training. During on-site clinical training, they exhibited ambivalent feelings, difficulty in suppressing their emotions to deal with the situation, and concerns that their relationships with others might be damaged if they expressed their feelings about sexuality [3]. Nursing techniques are not limited to the understanding and implementation of related procedures and necessary materials. In implementing such techniques, many other factors associated with providing nursing assistance are required, including an understanding of knowledge and meaning of the techniques, an understanding of the subject's condition, evaluation of the implementation results, and the need to practice with due consideration for safety and comfort.

To help novice learners acquire techniques for excretion assistance, it is necessary to clarify the specific challenges they face. According to the literature, four types of difficulties in genital care are identified: discrepancies between the implementation methods during actual clinical situations and those written in the textbooks; appropriate implementation in accordance with the patients' condition; few independent implementations of onsite clinical training; and confusion when taking care of male patients, due to lack of experience in practicing with male students during on-campus training [4]. In addition, students expressed more confusion when they provide nursing assistance during onsite clinical training than when they develop the nursing process and build nurse-patient relationships. They mentioned that this confusion was due to concerns about their first experience to put nursing technique into practice and as a result, they panicked and were unable to assist patients as they had expected [5]. Moreover, genital care and diaper changing are regarded as high-pressure nursing techniques, even though they are frequently implemented at on-site clinical training [6]. In light of the above mentioned situations, the following three factors are considered to be reasons that make it difficult for students to acquire such techniques: privacy problems specific to excretion assistance; the necessity of implementing nursing techniques with an understanding of the various factors associated with their implementation, which depend on patient situation; and difficulty in practicing excretion assistance due to the insufficient experience. Accordingly, we decided to implement a questionnaire survey to see how students acquire the excretion assistance techniques through on-campus training and on-site clinical training sessions for it so that we could clarify the specific points and degrees of difficulty for the nursing students.

When designing the questionnaire, the following literature sources were used for reference. In the "Study Group on the Modality of Nursing Education" of the Ministry of Education, Culture, Sports, Science and Technology, a report entitled "For improvement in fostering nursing practice competency in universities" was submitted in 2002 to present specific guidelines on the attainment targets of skills and techniques required for nursing students to become nursing professionals who are truly valuable for communities. In the guidelines, the "basic techniques of nursing," which is a basic technique for fostering practical competence, was integrated as a "learning technique for supporting nursing practice." In addition, components of attitudes and behavior that support the basic techniques of nursing were also specified [7]. Based on these components, a total of seventeen questionnaire items were included on the questionnaire survey form.

\section{Research Purpose}

The purpose of this study was to understand which nursing skills are easy to learn, and which are not at on-campus and on-site clinical training by conducting a questionnaire survey on the sense of learning achievement regarding excretion assistance techniques on-campus and on-site clinical training on excretion for junior nursing students learning such nursing techniques for the first time.

\section{Research Significance}

Although it is difficult for novice learners to acquire excretion assistance techniques under the current situation, the acquisition of solid technique is required. We can discuss the educational methods of nursing technique that allow nursing students to surely acquire excretion assistance techniques, and to develop an educational method that provides easy learning of such techniques, by clarifying the points and degrees of difficulty for nursing students in acquisition of excretion assistance technique.

\section{Methods}

Research Design

A quantitative and descriptive study.

\section{Research Subjects}

The study was conducted on 120 students who entered university from 2015 to 2016.

\section{Method of Implementation}

We conducted a self-administered, anonymous questionnaire surveys a total of 3 times, using our uniquely-developed 
questionnaire form. The first survey was conducted after the 1st grade students learned the assistance techniques for genital care and diaper changing in the second semester (from October to January). The second survey was conducted after the 2nd grade students learned assistance techniques for temporary urinary catheterization in the first semester (from April to July). The third survey was conducted after the 2 nd grade students completed onsite clinical training (September). The purpose of the study, along with methods and ethical considerations were explained to the student subjects at the same time. The questionnaire forms were then distributed, and each student was asked to post the form in a collection box after filling in it.

\section{Questionnaire Contents}

Regarding formulation of the questionnaire form, the contents of the questions were uniquely developed with reference to the components of the attitudes and behavior that support the basic techniques of nursing described in the report, "Study Group on the Modality of Nursing Education," issued by the Ministry of Education, Culture, Sports, Science and Technology in March 2002 [7]. Questionnaire survey forms 1 and 2 included questions on the following 13 items in total: acquisition of knowledge; understanding of the relationship with existing knowledge; understanding of the necessity and significance of assistance; understanding of the patient's thoughts and their application in the practice of assistance; preparation for assistance; implementation of assistance; cleanup after assistance; understanding of the patient's response (, or students who play the role of patients); evaluating the results of assistance; explanation in accordance with an understanding of the patient (case setting); attention to safety; comfortable assistance; and, securing privacy. Each question was evaluated via a 5-point Likert scale, from "Could do very well" to "Could not do at all." In questionnaire survey 3 , the students' levels of experience in excretion assistance at on-site clinical training were determined at the beginning. If students had experience in excretion assistance, they were then asked about their sense of learning achievement. In the questionnaire survey form, the items of preparation, implementation, and cleanup for assistance among the 13 items in questionnaire survey forms 1 and 2, were integrated as one topic. And 6 items including confirmation of instructions for excretion, judgement of the necessity of the instructions, implementation in accordance with individual situation, assistance for the family, explanation to the family, and consultation/advice/ guidance on self-care were added, resulting in a total of 17 items. The degree of achievement for each item was evaluated via a 6-point scale with "Not applicable," in addition to a 5-point Likert scale from "Could do very well" to "Could not do at all."

\section{Method of Analysis}

The answers were simply aggregated by question topic in questionnaire survey forms 1, 2, and 3. All collected questionnaire survey forms were subjected to analysis. In questionnaire survey 3 , only forms which students answered "yes" regarding experience in excretion assistance during their on-site clinical training were subject to analysis. As a questionnaire survey result, the percentages of sense of learning achievement for each item were calculated using the number of valid responses as the denominator. The answers "Could do very well" and "Could do" were regarded as "Could acquire the technique," and similarities and differences were compared and content discussed for items that exhibited high and low learning achievement percentages. Pearson's correlation coefficient was calculated to examine the relationships of each item. SPSS (Ver. 23) was used for statistical processing and significance level 0.01 was used.

\section{Ethical Considerations}

The purpose and method of study, optionality of participation, protection of personal information, and non-involvement of school performance and achievement were fully explained, and only students who gave informed consent joined the study. The collection box for the questionnaire survey forms was placed in a location different from the location for form completion so that individuals who filled out the questionnaire were not specified. The study was conducted with the approval of ethical review committee at the researchers' affiliation.

\section{Results}

Collection rates of questionnaire survey forms and number of valid responses (Table 1).

\begin{tabular}{|c|c|c|c|}
\hline & Survay 1 & Survay 2 & Survay 3 \\
\hline The collection rates & $49 \%$ & $41 \%$ & $34 \%$ \\
\hline The valid response rates & $100 \%$ & $94 \%$ & $89 \%$ \\
\hline
\end{tabular}

Table 1: The collection rates for the questionnaire survey forms and number of valid responses.

The collection rates for the questionnaire survey forms and number of valid responses are shown in Table 1. The collection rates were lower than $50 \%$ for all surveys. In addition, it was noted that the collection rates became lower as their grades advanced (Questionnaire survey 1: 49\%, Questionnaire survey 2: 41\%, Questionnaire survey 3: 37\%). The valid response rates were higher than $80 \%$ for all questionnaire survey forms.

\section{Questionnaire Survey Results}

Questionnaire Survey 1

In the second semester (from October to January) of the 1st grade, students were asked about their sense of achievement for genital care and diaper changing at on-campus training. Patient conditions were set up and students played the role of simulated patients in rotation. When they practiced genital care, a model of the genital area with simulated stool was used and the simulated patients wore a pair of leggings so that they would not reveal bare skin during the diaper changing.

The aggregated results for questionnaire survey 1 are shown in Figure 1. The highest sense of learning achievement was "understanding of the necessity and significance of assistance" (98.3\%), followed by "acquisition of knowledge" (96.5\%), and "attention to safety" (93\%). The lowest sense of learning achievement was "implementation of assistance" (73.7\%), followed by "understanding of the patient's response (with 
students playing the patients' role)" (79.0\%) and "explanation in accordance with an understanding of the patient (case setting)" $(80.7 \%)$. The percentages of learning achievement for items other than "implementation of assistance" and "understanding of the patient's response" were over $80 \%$, and students indicated that they had generally acquired all techniques for each item.

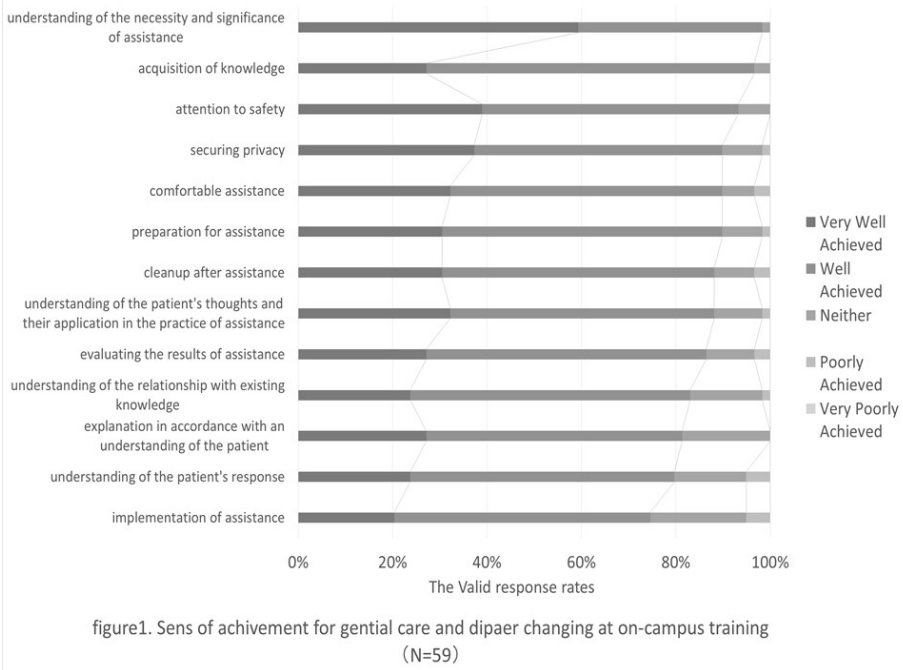

The correlation coefficients obtained from the questionnaire survey 1 are calculated. Strong positive correlations with "implementation of assistance" were found in 5 items: "acquisition of knowledge" $(\mathrm{r}=0.545)$; "preparation of assistance" $(\mathrm{r}=0.576)$; "attention to safety" ( $\mathrm{r}=0.365)$; "comfortable assistance" $(\mathrm{r}=0.441)$; and, "securing privacy" $(\mathrm{r}=0.436)$. Positive correlations were also found in "understanding of the patient's thoughts" $(r=0.295)$ and "understanding of the patient's response" $(\mathrm{r}=0.310)$.

\section{Questionnaire Survey 2}

In the first semester (from April to July) of the 2nd grade, students were asked about their sense of achievement regarding temporary urinary catheterization at on-campus training. Students were placed in situational settings and played the role of simulated patients in rotation. A model with only the genital area was used for urinary catheterization and training was conducted under the assumption that the patient excreted urine though simulated urine was not used then.

The aggregated results of questionnaire survey 2 are shown in Figure 2. The highest sense of learning achievement was indicated in 2 items; "understanding the necessity and significance of assistance" and "acquisition of knowledge" (97.7\%), followed by "preparation of assistance" (95.4\%), and "understanding of the patient's thoughts" and "attention to safety" (93.2\%). The lowest sense of learning achievement was "evaluation of the results of assistance" (70.4\%), followed by "understanding of the patient's response (with students playing the role of patients)" (77.3\%) and "implementation of assistance" (84.1\%). The percentages of learning achievement for items other than "evaluation of the results of assistance" and "understanding of the patient's response" were over $80 \%$ and students answered, as in questionnaire survey 1 , that they had generally acquired all techniques for these items.

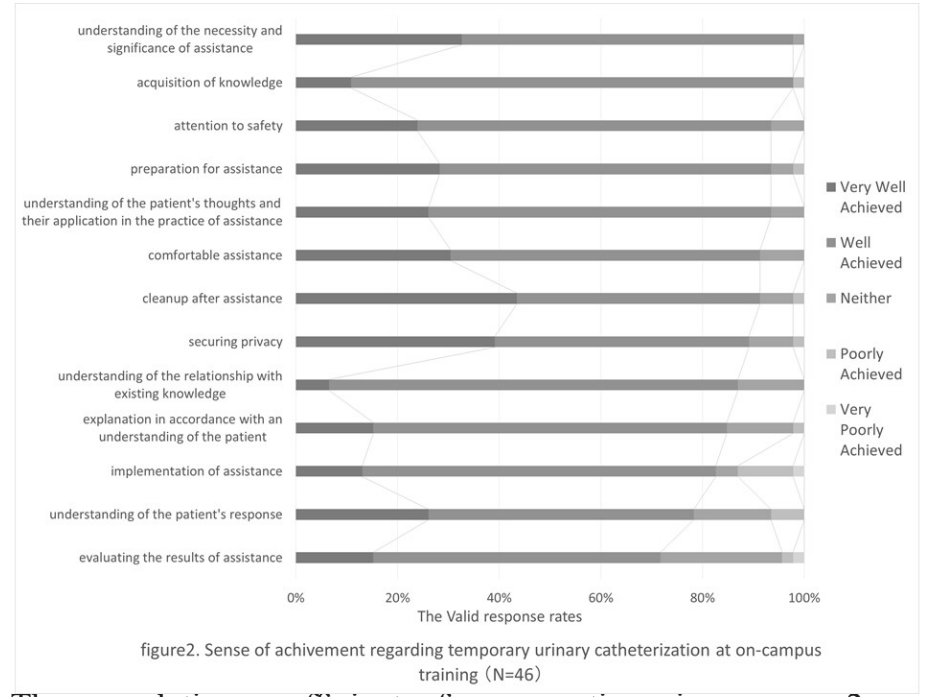

The correlation coefficients from questionnaire survey 2 are calculated. A positive correlation with "implementation of assistance" was found only in a single item, "acquisition of knowledge" $(\mathrm{r}=0.374)$. For urinary catheterization, simulated urine was not used and information about the amount and color of urine was shown only on paper. Therefore, it appeared that students felt it difficult to acquire techniques for "evaluation of the results of assistance."

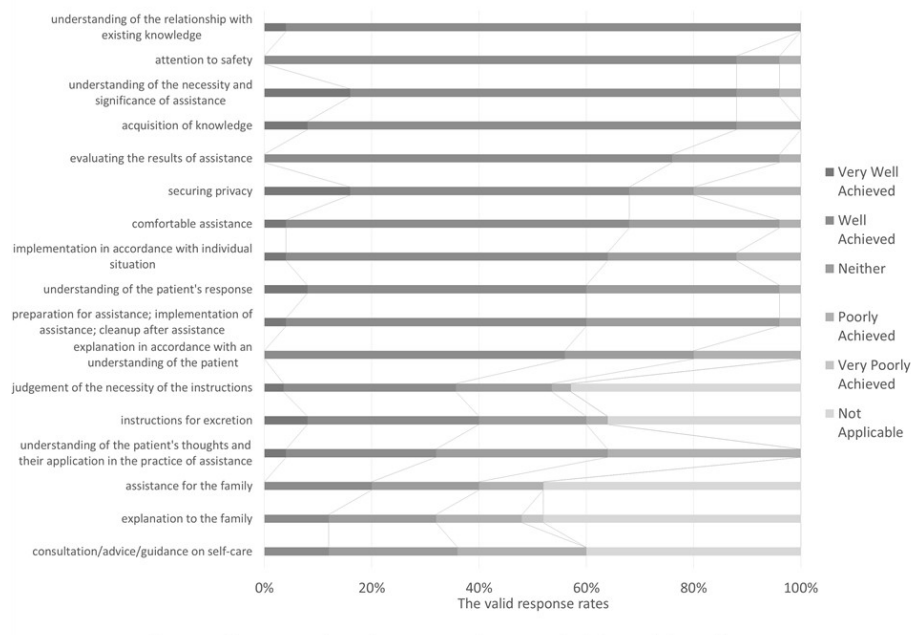

figure3. The on-site clinical training in the general adult ward $(N=26)$

\section{Questionnaire Survey 3}

In September, following completion of the lectures on basic nursing techniques of 2 nd grade students, the questionnaire survey was conducted after the on-site clinical training in the general adult ward of the university hospital. This was the on-site clinical training session in which the students were responsible for patients for the first time. These patients varied in disease and sex, and their ages were generally regarded as adult, although $80 \%$ were elderly persons.

Students were asked if they had received experience in excretion assistance in their on-site clinical training. The result showed that $68.2 \%$ of students had experience in excretion assistance. Students who answered that they had experience in excretion assistance were 
asked about their sense of its achievement. The highest sense of learning achievement was "understanding of the relationship with existing knowledge" (100\%), followed by "attention to safety," "understanding of the necessity and significance of assistance" and "acquisition of knowledge" (84.6\%). The lowest sense of learning achievement was "consultation/advice/guidance on selfcare" $(11.5 \%)$ and "explanation to the family about excretion assistance for the patient" (11.5\%), followed by "assistance for the family about excretion assistance for the patient" (19.2\%). All of these are items that were asked only in questionnaire survey 3 and $50-60 \%$ of students answered "not applicable." When the items were limited to the same as those employed in questionnaire surveys 1 and 2, the following results were obtained. The lowest sense of learning achievement was indicated for "understanding of the patient's thoughts and their application in the practice of assistance" (30.7\%), followed by "explanation in accordance with an understanding of the patients" (53.8\%), "preparation, implementation, and cleanup of assistance" and "assistance with understanding the patient's response" (57.7\%). In questionnaire survey 3 , the percentages of the sense of learning achievement were over $80 \%$ for only 4 of the above-described items which indicate a high sense of learning achievement. In questionnaire surveys 1 and 2, there were no items for which the percentages of learning achievement were lower than $70 \%$. In questionnaire survey 3 , the items for which the percentages of learning achievement were over $70 \%$ included only 5 items; "evaluation of the results of assistance" (73.1\%) in addition to 4 of the above-mentioned items.

The correlation coefficients from questionnaire survey 3 are calculated. Positive correlations with "preparation, implementation, and cleanup of assistance" were found in 4 items: "understanding the necessity and significance of assistance" ( $\mathrm{r}=0.400)$; "understanding of the patient's thoughts" $(\mathrm{r}=0.417)$; "attention to safety" $(\mathrm{r}=0.464)$; and, "securing privacy" $(\mathrm{r}=0.415)$.

ber of valid responses.

\section{Discussion}

Sense of learning achievement for excretion assistance

Regarding the learning achievement for excretion assistance, it was found that it was easy for students to understand the knowledge and significance of excretion assistance and to pay attention to safety. However, it was difficult for them to acquire practical techniques for implementing assistance and understanding patient responses. This finding is based on the following two observations. A higher percentage of students answered that they could acquire nursing techniques for three items; "understanding the necessity and significance of assistance," "acquisition of knowledge," and "attention to safety" in all three questionnaire surveys (1,2 and 3 ). On the other hand, a lower percentage of students answered that they could acquire nursing techniques for "implementation of assistance," "assistance with an understanding of the patient's response," and "explanation in accordance with an understanding of the patient." Implementing assistance in accordance with individual patient's situation, rather than in a systematically determined manner is a highly sophisticated technique for novice learners. In the feedback of OSCE, students also commented that "it is difficult to make judgments in accordance with individual patient's situation" and "it is difficult to accommodate myself to the patient" [8]. The results also indicate that those items for which the students have little confidence in their techniques despite their on-site clinical training experience include technical content "in accordance with the patient's condition" or with the assumption of a broader context [9]. Because repeated practice and the accumulation of experience are needed to give assistance with an understanding of patient responses and in accordance with the situation, it is difficult for novice learners with little experience to acquire these techniques. In particular, students are regarded to have less experience in excretion assistance than in meal assistance or hygiene assistance [2], and the sense of learning achievement could be lower in an understanding of patient response, which requires more experience. Providing more opportunities for students to gain experience is required to help them acquire techniques for the implementation of assistance with an understanding of patient responses. This is clear from the statement that the number of experiences is proportional to the degree of achievement, regarding the degree of achievement for nursing techniques in nursing students [1]. Students should gain experience without missing opportunities to have practical experiences in excretion assistance.

Comparison of Technique Acquisition at on-site Clinical Training and Campus Training

It is shown that students felt that it was more difficult to consider privacy, provide comfortable assistance, and implement assistance with an understanding of patient thoughts during on-site clinical training, compared to on-campus training. This finding is based on the following two observations. In questionnaire survey 3 , the lowest percentage of students answered that they were able to implement assistance while "understanding the patient's thoughts" $(30.7 \%)$, and percentages were also low for "comfortable assistance" (69.2\%) and "securing privacy" (65.4\%). On the other hand, in questionnaire surveys 1 and 2, relatively higher percentages of students (i.e., $80-90 \%$ ) answered that they were able to acquire techniques regarding "understanding of patients' thoughts" (questionnaire 1: 87.7\%, questionnaire 2: 83.1\%), "securing privacy" (questionnaire 1: $87.7 \%$, questionnaire 2:83.1\%), and "comfortable assistance" (questionnaire 1: 88.4\%, questionnaire 2: 90.9\%). The sense of learning achievement in on-site clinical training was lower in securing privacy, which is important in excretion assistance. As it is necessary to secure privacy in accordance with the environment and embarrassment of each patient in on-site clinical training, it is difficult for students to acquire techniques for securing privacy in on-site clinical training. On the other hand, at on-campus training, simulated patients are considered so that they would not reveal bare skin and students participate in role-play while imagining the situation of the patients. Therefore, privacy is rarely undermined at on-campus training and it appears that students are likely to feel that they could acquire the techniques for securing privacy.

At the on-campus training, for physically less-invasive modes of assistance such as genital care and diaper changing, it is important 
to give assistance with an understanding of the patient's response, provide safe and comfortable assistance, and provide appropriate assistance for the patient, including securing privacy. For highly invasive modes of assistance such as temporary catheterization, much knowledge is required; including the sterilization procedure and catheter insertion depth, acquisition of knowledge is needed together with the implementation of assistance. In on-site clinical training, it appears to be difficult for students to assist with an understanding of the patient's response, even when they provide physically less-invasive modes of assistance. This is clear from the following results. Considering the correlation coefficients from questionnaire survey 1 , the contents showing a positive correlation with "implementation of assistance" include "assistance with an understanding of the patient's response," "attention to safety," "comfortable assistance," and "securing privacy." Therefore, it appears that students can acquire techniques for assistance with an understanding of the patient's response, as well as safe and comfortable assistance while securing privacy when providing physically less-invasive modes of assistance. Considering the correlation coefficients from questionnaire survey 2, only "acquisition of knowledge" showed a positive correlation with "implementation of assistance."

Therefore, it appears that students should have more knowledge to assist when providing highly invasive modes of assistance. In on-site clinical training, most assistance involves physically lessinvasive modes, including genital care, diaper changing, and toilet assistance. In questionnaire survey 3 , the items that showed a positive correlation with "implementation of assistance" included "attention to safety," "assurance of privacy," "understanding of the necessity and significance of assistance," and "understanding the patient's thoughts." Based on this result, it appears that students can consider safety and secure privacy when assisting and ; however, it appears difficult for students to assist with an understanding of patient responses, and in consideration of the patients' situation. It is considered that the items that students feel difficult to perform during on-site clinical training involve the implementation of nursing assistance. In addition, students are likely to have worries about their first experience of any assistance and may often feel fear due to the presence of subject patients differing from those at on-campus training [5].

It appears that students are greatly challenged when assisting, and it is difficult for them to establish a comfort zone where they can feel relaxed in understanding of patient responses while providing excretion assistance. It is said that although confidence in nursing technique is proportional to the number of experiences, those items which students have little confidence in their technique, despite their elevated level of experience often corresponds to those including technical content "in accordance with the patient's condition" or with the assumption of broader context [9]. In onsite clinical training, it is significantly difficult for students to provide excretion assistance with an understanding of the patients' situations and it will continue to be a challenge, even if the experience level of the students is improved.
Discussion on the Educational Method for Acquiring Techniques in Excretion Assistance

Acquiring an understanding of knowledge and the significance of assisting is easy in both on-campus and on-site clinical training. In addition, it is easier to associate with existing knowledge in on-site clinical training. Therefore, as such an understanding can be fully acquired during on-campus training, it is necessary to improve the learning environment so that it can be surely acquired by students. Moreover, because it is easy for students to acquire an understanding of the knowledge and significance of assistance in on-site clinical training, it may be effective to facilitate an understanding of such knowledge. Association with existing knowledge is also easy to acquire in on-site clinical training, possibly because the knowledge and significance learned by taking various courses become integrated into the student through the act of being responsible for a patient. Therefore, it appears to be effective to give instructions to students so that they can associate the content of training with existing knowledge in on-site clinical training.

At on-campus training, skill in assisting in accordance with patients' responses is easy to acquire for physically less-invasive modes of assistance, however, is difficult to acquire for highly invasive modes. In addition, in on-site clinical training, where students take charge of patients, it is difficult to acquire such skill, even if it is a physically less-invasive assistance. Therefore, the use of ingenuity is recommended during on-campus training, including having students deliberate about what kind of assistance is needed in accordance with the patient setting and giving commentaries on such points. By changing the content of on-campus training to have more role-plays, the implementation rates for techniques that previously students had only watched have been improved [10]. And experiences of role-plays even at on-campus training may lead to an improvement in the implementation of assistance in onsite clinical training. In on-site clinical training, it is necessary to offer more opportunities to gain experience in excretion assistance. Actual experience helps students reduce the initial confusion or fear regarding the implementation of assistance techniques and helps them to turn their attention to understand patients' responses. With ingenuity in increasing opportunities to gain experience in implementing techniques during on-site clinical training, the implementation rates for techniques that previously students had only watched have been improved [10].

It is necessary to improve the practical training environment so that students may gain more experience in nursing techniques. In addition to have students more opportunities to gain experience, instructions for them to turn their attention to implementing assistance with an understanding of patients' responses as well as careful feedback on how students have incorporated responses from patients into the assistance may lead to improved practice for subsequent cases. Looking back on on-site clinical training helps students to review their own care and may lead to practice for subsequent cases [11].

When teachers assist patients with students, the understanding 
of the students may also be improved by reflecting with them on how well they have understood the patients' responses, and how that led to the implemented assistance. It is mentioned that timely feedback from teachers allows students to become aware of experiences during the implementation of assistance, resulting in an improvement of their technical experience level [12]. In addition, it is also considered that reflection by novice nurses may deepen their understanding of practice and may have a favorable effect on subsequent practice [11]. Solid reflection on how students understand patient responses and how such responses are reflected into their assistance in accordance with the patients' situations is effective in helping students to acquire skill in implementing assistance with an understanding of patients' responses.

\section{Study Limitations}

As this is a questionnaire survey study conducted only at a single facility, the results are difficult to generalize. In addition, as the collection rates of questionnaire forms were below $50 \%$, it is difficult to establish that the results reflect the general population. Accordingly, further questionnaire surveys at multiple facilities are required in the future. In addition, methods to improve the questionnaire form collection rate should be reexamined.

\section{Conclusions}

- Regarding excretion assistance, students feel that it is easy to acquire an understanding of knowledge and the significance of assistance as well as attention to safety. However, students feel that it is difficult to acquire skill in implementing assistance and assisting while considering patients' responses.

- Students have a generally higher sense of learning achievement for excretion assistance at on-campus training. In on-site clinical training, they have a higher sense of learning achievement for acquiring an understanding of knowledge and the significance of assistance as well as relating such an understanding with existing knowledge, but have a lower sense of learning achievement for other items.

- "Understanding of patient thoughts," Securing privacy," and "comfortable assistance" are the skills students felt easy to acquire at on-campus training. However, they felt those are difficult in on-site clinical training.

- At on-campus training, students can acquire techniques for assistance with an understanding of patient responses, and in consideration of safety and comfort when they implement physically less-invasive modes of assistance, but they focus on the acquisition of knowledge during highly invasive modes of assistance.

- In on-site clinical training, students can think about safety and the securing privacy while assisting; however, it is difficult for them to assist with an understanding of patient responses.

- Because it is difficult for students to assist with an understanding of patient responses in on-site clinical training, educational guidance to allow students to fully learn techniques at oncampus training and to acquire further techniques in on-site clinical training is required.

\section{Reference}

1. Oriyama S, Okamoto A. The Status of Experiences of Nursing Skills in Clinical Practice among Nursing Students and Factors Influencing the Subjective Attainment Level An Analysis of Several Nursing Education Institutions in the Chugoku Region. Japan Academy of Nursing Science. 2015; 35: 127-135.

2. Shimizu Y, Hamano Y, kawanami K. The acquired nursing techniques of nutrition care, elimination care and hygienic care at the time of employment of novice nurse. Journal of Japan Nursing Society Care Management. 2014; 44: 277-280.

3. Mizuno M, Fukuda H. The educational current state and problem about sexuality in genital cleaning of a male patient. The Japanese Journal of Nursing Education. 2010; 51: 134139.

4. Hayakawa M, Nakamura K, Furuta M. A Literature Review on Teaching Methods of Private Parts Washing in Basic Nursing Education. The Journal of Chukyo Gakuin University School of Nursing. 2014; 4: 75-46.

5. Nakamoto A, Ito A, Yamamoto J, et al. Characteristic of difficult feelings that nursing students hold and comparison according to situation in Clinical Practice of Nursing; Comparison between Fundamental Nursing Clinical Practice and Adult Nursing Clinical Practice. The Journal of Senrikinran University. 2015; 12: 123-134.

6. Oku Y, Daizou M, Kaganomiya Y, et al. Study on Nursing Students Strain of Nursing Techniques in Clinical Practice. Bulletin of Gifu University of Medical Science. 2012; 6: 121127.

7. http://www.umin.ac.jp/kango/kyouiku/report.pdf.

8. Yasui D, Ozawa T, Hamada M, et al. Students learning and Discovery through Reflection conducted of Post-operative early ambulation care OSCE. Bulletin of Tokyo Healthcare University. 2014; 1: 23-30.

9. Katahira N, Koyama M, Ohishi T, et al. Nursing Students Experience and Confidence about Nursing Skills in Clinical Practice in Japan. Japan Academic Nursing Education. 2012; 22: 65-71.

10. Hasegawa Y, Saitoh H. Effect of Practical Training and Campus Practice to Improve Nursing Skills among Students in a Pediatric Nursing Practicum. Japan Academic Nursing Education. 2016; 26: 89-96.

11. Lee H, Fukada A, Shinbashi S, et al. Content analysis of clinical training reports after the incorporation of portfoliobased learning: Introspection on clinical training among nursing students. Japanese Journal of Nursing and Health Sciences. 2016; 14: 20-13.

12. Ogiwara M, Saito T, Ychi W, et al. Research on actual conditions for nursing-skills experience in adult nursing clinical practices Comparison with pilot study. Bulletin of Japanese Red Cross Akita College of Nursing. 2015; 20: 25-34.

(C) 2018 Noriko A. This article is distributed under the terms of the Creative Commons Attribution 4.0 International License 